\title{
Linx
}

Revue des linguistes de l'université Paris X Nanterre

$54 \mid 2006$

La cause : approche pluridisciplinaire

\section{Les quatre questions de Tinbergen}

\section{Mathieu Amy}

\section{OpenEdition}

\section{Journals}

Édition électronique

URL : http://journals.openedition.org/linx/499

DOI : $10.4000 /$ linx.499

ISSN : 2118-9692

\section{Éditeur}

Presses universitaires de Paris Nanterre

\section{Édition imprimée}

Date de publication : 1 juin 2006

Pagination : 27-33

ISSN : 0246-8743

\section{Référence électronique}

Mathieu Amy, "Les quatre questions de Tinbergen », Linx [En ligne], 54 | 2006, mis en ligne le 01 août 2007, consulté le 19 avril 2019. URL : http://journals.openedition.org/linx/499; DOI : 10.4000/linx.499 


\title{
Les quatre questions de Tinbergen
}

\author{
Mathieu Amy, Université de Paris X-Nanterre, \\ Ethologie et Cognition Comparées (EA 3456)
}

\section{Introduction}

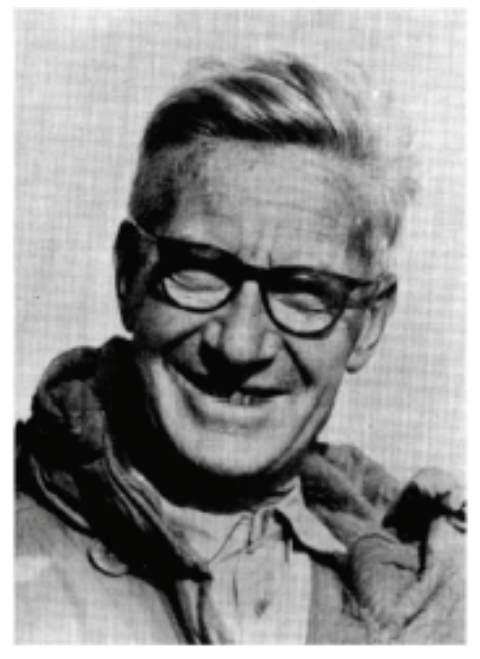

Nikolaus Tinbergen est né en 1907 aux PaysBas dans la ville de La Haye. Il étudia la biologie à l'université de Leiden, et y soutint sa thèse en 1932. Tinbergen partit en 1933 pour une expédition au Groenland puis revint travailler aux Pays-Bas en tant qu'assistant au département de zoologie. Après la seconde guerre mondiale, durant laquelle il fut emprisonné dans un camp de travail, il devint professeur de zoologie à l'université de Leiden. En 1949 il devint maitre de conférence à l'université d'Oxford où il fonda le Groupe de recherches en Comportement animal. Tinbergen mourut en 1989, laissant derrière lui un travail considérable avec plus de trois cents publications, dont l'article «On aims and methods of ethology » de 1963 qui formalisa les grandes questions de l'éthologie.

Étymologiquement, le terme éthologie signifie «discours sur les mœurs», «science du comportement», aujourd'hui l'éthologie est définie comme l'étude du comportement animal. Le comportement peut être défini comme l'ensemble des mouvements objectivables exécutés par un individu (Bovet, 1964). Globalement 
l'éthologie cherche à dégager des lois pour expliquer les comportements les plus simples comme les plus élaborés.

Les deux «fondateurs » de l'éthologie sont Konrad Lorenz (1903-1989), autrichien, connu pour ses travaux sur l'empreinte chez les oies cendrées, et Nico Tinbergen. Tous deux reçurent le Prix Nobel de médecine en 1973 avec Karl von Frisch (18861983), autrichien, connu notamment pour ses travaux sur la communication chez les abeilles (Frisch, 1927). Ils représentaient l'école objectiviste de forte influence naturaliste et ont tous trois contribué à poser les bases de l'éthologie classique.

Lorenz fut le premier à considérer que les comportements, et donc les mécanismes qui les sous-tendent, sont adaptés de la même façon que les organes le sont (Lorenz, 1937). Les comportements sont donc le fruit de l'évolution, ils ont certaines fonctions qui permettent aux animaux de survivre et de se reproduire. Ainsi chaque espèce a des comportements qui lui sont propres. Une grande partie des premiers travaux en éthologie a été de répertorier et de décrire les comportements communs à tous les individus d'une même espèce. Ce répertoire comportemental est appelé un éthogramme. Les traits comportementaux contribuent ainsi à la classification des espèces. Chaque espèce a donc des comportements qui lui sont propres mais, nous le verrons par la suite, ce n'est pas pour autant qu'ils sont innés et qu'ils ne nécessitent pas d'apprentissage. Néanmoins, cette conception de comportements propres à une espèce a amené les éthologistes à utiliser les concepts d'« instinct » et de «comportement inné », qui alimentèrent le débat nature-culture entre les courants béhavioristes et éthologistes (objectivistes).

Nous proposons de présenter un article fondateur de Nico Tinbergen publié en 1963 et qui s'intitule " On aims and methods of ethology », que l'on peut traduire par

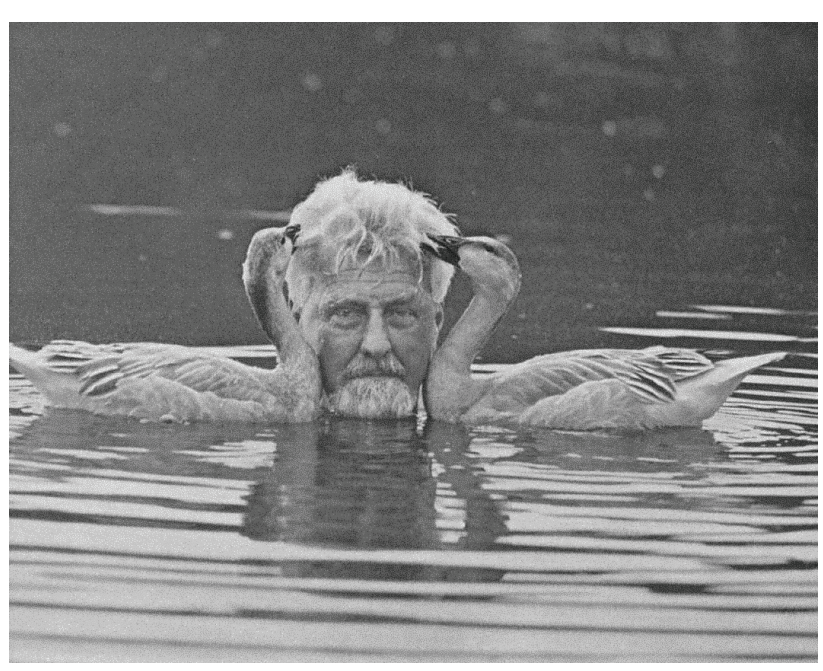
« des buts et des méthodes de l'éthologie » (Tinbergen, 1963). Tinbergen a écrit cet article pour plusieurs raisons. D'abord, il souhaitait se dégager de l'image de l'éthologie vis-àvis du grand public. L'éthologie était perçue comme un passetemps pour de grands enfants. La photo ci-contre montrant Lorenz se baignant avec deux oies imprégnées participe à cette image. De plus, la mise en place d'éthogrammes nécessite de passer beaucoup de temps à observer les animaux dans la nature.

Bien qu'étant des contributions modestes car descriptives, les éthogrammes sont, pour Tinbergen, un complément indispensable à la méthode expérimentale (Tinbergen, 1963). Pourtant le grand public avait peut-être beaucoup de mal à juger crédible des chercheurs passant leur temps dans la nature, plutôt qu'en blouse blanche dans un 
laboratoire. Dix ans plus tard, l'éthologie avait encore du mal à se dégager de certains clichés comme celui de l'anthropomorphisme (Lorenz, 1973).

Ensuite Tinbergen (1963) rappelle que l'éthologie est née aussi en réaction contre la psychologie expérimentale américaine appelée «béhaviorisme ». Les éthologistes avaient alors fait essentiellement deux reproches aux béhavioristes. Le premier est que les animaux, dans la nature, ne sont jamais soumis aux conditions qu'ils rencontrent dans les situations expérimentales et que, par conséquent, les comportements qu'étudient les béhavioristes ne sont ni normaux, ni naturels. Le second reproche est que les béhavioristes avaient tendance à considérer que les résultats obtenus sur une espèce donnée sont généralisables à toutes les autres espèces. D'ailleurs Skinner disait «Pigeon, rat ou singe, lequel est lequel ? Ça n’a pas d'importance» (voir Barlow, 1991). Les béhavioristes ne se souciant pas de ce qui se passe «à l'intérieur » de l'animal, ils négligeaient aussi les différences des systèmes nerveux entre espèces. Néanmoins les éthologistes ont salué la valeur des travaux des béhavioristes qui nous renseignent sur les facultés d'apprentissage des animaux.

Enfin la troisième et principale raison pour laquelle Tinbergen a écrit cet article était de clarifier la nature des questions posées par l'éthologie.

Pour cela Tinbergen insiste sur la filiation qui existe entre la biologie et l'éthologie. La théorie de l'évolution de Darwin et ses processus de sélection naturelle (Darwin, 1859) et de sélection sexuelle (Darwin, 1877) sont donc essentiels à l'éthologie.

Tinbergen nous explique aussi que l'éthologie se pose les mêmes types de questions que la biologie et reprend les trois problèmes majeurs dictés par Huxley : celui de la causalité, celui de la valeur de survie et celui de l'évolution. A ces trois problèmes, Tinbergen en rajoute un quatrième : celui de l'ontogenèse. Ces quatre problèmes sont évidement à l'origine des quatre questions de Tinbergen, à savoir :

1) Quelles sont les causes proximales des comportements ?

2) Quelles sont les causes ultimes des comportements?

3) Quelle est l'ontogenèse des comportements ?

4) Quelle est la phylogenèse des comportements ?

\section{Les quatre questions de Tinbergen}

1) Quelles sont les causes proximales des comportements ? En d'autres termes, quels sont les facteurs qui déclenchent un comportement ? Ici il convient de distinguer les facteurs externes des facteurs internes :

- les facteurs externes sont toute modification du milieu susceptible de faire réagir l'animal de façon spécifique à cette modification ;

- les facteurs internes sont, toute modification de l'organisme jouant un rôle dans le déclenchement du comportement étudié, d'où l'importance de connaitre les systèmes sensoriels, nerveux, hormonaux et musculaires de l'espèce étudiée pour expliquer ses comportements.

Prenons un exemple: quels sont les causes proximales du chant d'un oiseau mâle ? En d'autres termes quels sont les facteurs qui font qu'un oiseau mâle se mette à 
chanter? Pour schématiser, on sait que la vue d'une femelle ou le fait d'entendre un autre mâle chanter vont inciter le mâle à chanter. Ces deux facteurs sont donc des facteurs externes. On sait aussi que certaines structures nerveuses et hormonales jouent un rôle essentiel dans le déclenchement du chant d'un mâle. Cela fait donc référence à des facteurs internes. Ainsi un noyau neuronal appelé «High vocal center » (HVC) est indispensable chez les oiseaux pour pouvoir chanter. Le comportement de chanter est évidemment bien plus que la stimulation directe de l'HVC sur les tissus permettant de chanter mais cette structure reste nécessaire. Certains facteurs externes et internes sont intimement liés car des modifications de l'environnement vont entraîner des modifications de l'organisme qui, à leur tour, vont être propices au déclenchement d'un comportement.

2) Quelles sont les causes ultimes des comportements ? En d'autres termes quelle est la fonction d'un comportement donné, à quoi sert-il ? Quelle est la valeur d'un comportement pour la survie d'un individu? Si l'on reprend l'analogie que Lorenz fait entre les comportements et les organes, les comportements ont des fonctions, comme les organes en ont, et les comportements vont donc garantir l'adaptation de l'individu à son milieu, par exemple pour s'alimenter, se reproduire, fuir ses prédateurs ou encore réguler ses comportements sociaux.

Reprenons notre exemple du chant des oiseaux : pourquoi les oiseaux chantentils? Quelle est la fonction du chant? D'abord tous les oiseaux ne chantent pas. Les espèces qui chantent sont appelées «oscines». Et souvent seuls les mâles chantent chez ces espèces. Il est admis deux grandes fonctions au chant des mâles (voir Nowicki \& Searcy, 2005). La première fonction est la défense d'un territoire : en chantant, les mâles signalent leur présence à d'éventuels rivaux. La seconde fonction est l'attraction du partenaire, c'est-à-dire l'attraction d'une femelle de son espèce dans le but de se reproduire avec elle.

3) Quelle est l'ontogenèse des comportements ? C'est-à-dire, comment un comportement apparait-il au cours de la vie d'un individu ? Quels sont les rôles de l'environnement et des prédispositions dans l'apparition d'un comportement?

Dans l'exemple du chant des oiseaux: les oiseaux apprennent-ils à chanter? Si oui, comment? On sait que chez de nombreuses espèces, cet apprentissage est indispensable pour que le chant devienne fonctionnel (défendre son territoire et surtout attirer les femelles). Durant cet apprentissage, on peut distinguer une période d'acquisition qui précède une période d'imitation. Durant la période d'acquisition, l'oiseau apprend les chants de tuteurs (préférentiellement vivants et de leur espèce). Durant la période d'imitation, le mâle "s'entraîne » à chanter jusqu'à ce que son chant devienne fonctionnel. Selon les espèces ces deux périodes se chevauchent ou non dans le temps.

4) Quelle est la phylogenèse des comportements ? Comment un comportement est apparu au cours de l'évolution?

Cette question est certainement la plus difficile car, contrairement aux paléontologues qui peuvent «remonter le temps » grâce aux ossements, on ne peut que constater en éthologie les comportements d'espèces toujours vivantes. Pour pallier 
cette difficulté une méthode consiste donc à comparer les comportements d'espèces proches et d'inférer que des comportements assez similaires pouvaient appartenir à une espèce ancestrale. Ce qui est important de comprendre ici, c'est que pour qu'une évolution des comportements s'établisse, il doit d'abord exister une variation des comportements chez l'espèce ancestrale. Le processus de spéciation se fera donc à partir de ces divergences, et les espèces qui découleront de l'espèce ancestrale auront des comportements qui se ressembleront, mais qui seront à la fois suffisamment différents pour constituer un isolement reproducteur entre ces espèces proches.

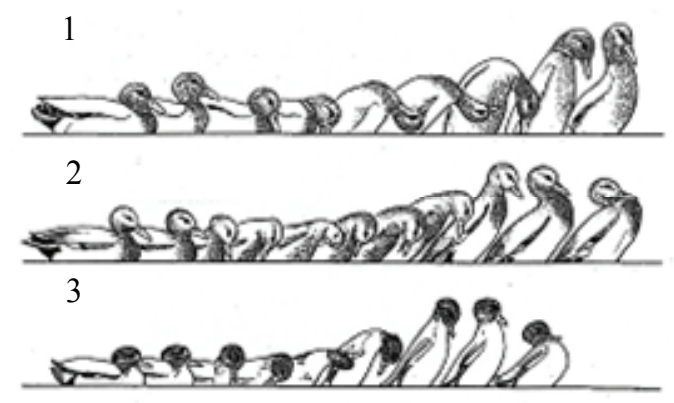

Prenons un exemple plus visuel que le chant des oiseaux. Sur l'image ci-contre, nous pouvons voir le comportement de parade des mâles de trois espèces différentes de canard (de haut en bas : le canard colvert 1 , le canard chipeau 2 et la sarcelle d'hiver 3). Globalement ces trois parades se ressemblent: l'oiseau se tient sur l'eau, baisse la tête puis la remonte en faisant sortir son ventre de l'eau. Ces similitudes laisse penser que cette parade faisait partie du comportement d'une espèce ancestrale commune à ces trois espèces.

\section{La notion de « cause » selon Tinbergen}

Selon Tinbergen (1963), l'étude de la causalité en éthologie est l'étude des événements antérieurs dont on peut montrer qu'ils contribuent à l'occurrence d'un comportement. Les effets (ici les comportements) sont observés et les causes inférées. Mais les comportements observés ont également des effets sur la survie de l'individu. Le comportement observé devient alors la cause de la survie (effet) de l'individu. Selon Tinbergen (1963), cette distinction entre causes et effets est due à la perception humaine : nous arrivons à observer un comportement plus facilement que la survie et c'est pourquoi nous prenons un point arbitraire parmi la succession des événements.

Tinbergen (1963) écarte également toute explication d'ordre téléologique comme cause possible du comportement des animaux.

\section{L'éthologie « moderne»}

Même si l'étude du comportement animal a évolué depuis que Tinbergen a formulé ses quatre questions, ces dernières restent néanmoins sous-jacentes aux problèmes des éthologistes d'aujourd'hui. L'éthologie «classique» de Lorenz, Tinbergen et Von Frisch a donné naissance à diverses sous-disciplines que sont principalement l'éthologie cognitive, l'écologie comportementale et la sociobiologie. D’une façon générale ces 
courants ont été enrichis par d'autres disciplines et mettent l'accent sur certaines questions plutôt que d'autres.

L'éthologie classique, c'est-à-dire telle qu'elle était pratiquée dans les années soixante-dix, a peu étudié les différences entre les individus d'une même espèce. Pourtant, comme nous l'avons vu plus haut, cette variabilité inter-individuelle est nécessaire à une évolution des espèces. Le courant de l'écologie comportementale (Behavioural Ecology, Krebs et Davies, 1981) prend en compte la diversité des individus au sein d'une espèce. Selon cette discipline les individus gèrent des budgets temps-énergie pour maximiser les bénéfices et minimiser les coûts, et certains individus sont mieux adaptés que d'autres pour gérer ces budgets. L'écologie comportementale met l'accent sur les causes ultimes et évolutionnistes des comportements et sur les facteurs environnementaux.

Une autre discipline insiste sur l'approche évolutive du comportement grâce notamment à la place centrale qui est attribuée aux gènes. Selon la sociobiologie et la sélection de parentèle de Wilson (1975), « l'individu n'assure pas sa reproduction mais est au service de ses gènes qui cherchent à travers lui à passer à la génération suivante ou à favoriser le passage de gènes similaires, tout d'abord ceux d'individus apparentés » (Kreutzer \& Vauclair, 2004). Cette nouvelle approche a permis de répondre à des questions longtemps restées en suspens comme l'existence d'individus stériles aidant un seul congénère à se reproduire chez les insectes sociaux.

Enfin l'éthologie cognitive (terme inventé par Griffin en 1976) intègre des concepts venant de la psychologie et des neurosciences en général. Ce courant de l'éthologie couvre un vaste champ de recherches et va particulièrement privilégier les mécanismes proximaux internes grâce à l'étude des structures nerveuses impliquées dans les comportements. L'ontogenèse des comportements y tient également une place prépondérante. 


\section{BIBLIOGRAPHIE}

BARLOW, G.W., 1991, «Nature-Nurture and the Debates Surroundind Ethology and Sociobiology ». American Zoologist 31: 286-296.

Bovet, J., 17 Janvier 1964, Position de l'Ethologie au sein des sciences du comportement. Leçon inaugurale prononcée à l'Université de Lausanne.

DARWIN, C., 1859, On the Origin of Species by Means of Natural Selection. Londres, John Murray.

Darwin, C., 1877, The Descent of Man and Selection in Relation to Sex. Londres, John Murray.

FriscH, K.v., 1927, Aus dem leben der bienen. Springer-Verlag OHG. Berlin.

GrIFFIN, D.R., 1976, The Question of Animal Awareness : Evolutionary Continuity of Mental Experience. New York, Rockefeller University Press.

KREBS, J.R. \& Davies, N.B., 1978, Behavioural ecology: An Evolutionary Approach. Oxford, Blackwell Scientific Publication.

Kreutzer, M. \& VAuclair, J., 2004, La cognition animale au Carrefour de l'éthologie et de la psychologie. Paris : Editions La Maison des Sciences de l'Homme.

LorenZ, K.Z., 1937, «Uber die Bildung des Instinktbegriffes ». Naturwiss. 25 : 289-300, 307318, 324-331.

LORENZ, K.Z., December 12, 1973, Analogy as a source of knowledge. Nobel Lecture.

NOWICKI, S. \& SEARCY, W.A., 2005, Song and mate choice in birds : how the development of behaviour helps us understand function. The Auk 122 (1): 1-14.

Tinbergen, N., 1963, «On aims and methods of Ethology ». Zeitschrift für Tierpsychologie, 20, 410-433.

WiLsOn, E.O., 1975, Sociobiology. Cambridge MA, Harvard University Press.

\section{Photographies}

Photo de Tinbergen: Peter MARLER, 2005. Ethology and the origins of behavioral endocrinology. Hormone \& Behavior 47 : 493-502.

Photo de Lorenz : Life, 1966.

Parade des canards : Mark RIDLEY, 1995. Animal Behavior. An introduction to behavioral mechanisms, development, and ecology. Second edition. Blackwell Scientific Publications, Boston, 288 p. 
